\title{
NOUVELLe
}

\section{Microrégulation aux frontières (cérébrales)}

Marion Coolen, Laure Bally-Cuif
Helmholtz Zentrum München, Department Zebrafish Neurogenetics, Institute of Developmental Genetics, Ingolstaedter Landstrasse 1, 86764 Neuherberg, Allemagne. marion.coolen@helmholtz-muenchen.de bally@helmholtz-muenchen.de

\section{Régionalisation et frontières}

\section{au cours du développement cérébral}

Le développement du système nerveux central des vertébrés repose sur deux processus fondamentaux : la régionalisation, qui attribue à chaque territoire une identité propre, et la neurogenèse, qui contrôle l'émergence de cellules neurales différenciées fonctionnelles (neurones et glies) à partir des cellules progénitrices [1]. Chez l'embryon, la coordination de ces processus est en partie permise par l'établissement au sein du tube neural de frontières organisatrices [2]. Par l'intermédiaire de molécules de signalisation morphogènes, ces frontières déterminent l'identité de leurs territoires voisins. Fréquemment, elles servent simultanément de centres de contrôle de la neurogenèse, par exemple en abritant pendant de longues durées des cellules progénitrices. Les mécanismes moléculaires assurant la coordination spatiale de ces deux activités restent mal connus.

Une frontière très étudiée est la MHB (midbrain-hindbrain boundary), sépa- rant cerveau moyen (mésencéphale) et cerveau postérieur (rhombencéphale). La MHB contrôle la régionalisation du mésencéphale et du rhombencéphale antérieur grâce à la voie de signalisation Fgf, en particulier au signal Fgf8, à son récepteur Fgfrl, et à l'activateur Canopyl [3] (Figure 1A). La MHB est également un réservoir de cellules progénitrices, dont le recrutement progressif assure la neurogenèse més- et rhombencéphalique. Le maintien des cellules progénitrices de la MHB dépend de facteurs de transcription de type Hairy/E(Spl) (hairy/enhancer of split) : Hes (Hesl/3/5) chez la souris, Her (Her5/9) chez Danio [4] (Figure 1B). Afin d'assurer la cohérence des fonctions de la $M H B$, il est important que l'expression des facteurs Fgf et Hes/Her soit maintenue en phase après leur induction initiale. Nous avons recherché des régulateurs potentiels parmi les microARN, petits ARN noncodants de 20-22 nucléotides qui modulent la traduction de familles d'ARNm cibles par l'intermédiaire de séquences 3’UTR spécifiques [5, 12].

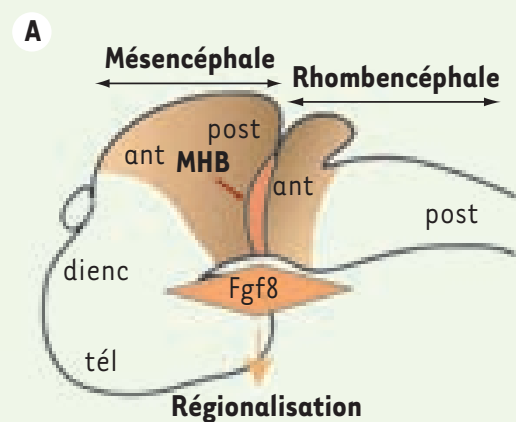

B

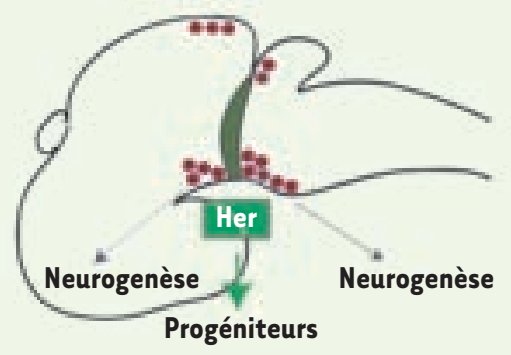

miR-9 est essentiel

\section{à la restriction spatiale de la MHB}

Différents éléments nous ont conduits à mener une investigation poussée du microARN miR-9 [6]. En premier lieu, des recherches in silico de sites de liaison de microARN nous ont permis de détecter des sites susceptibles de lier miR-9 sur les régions 3'UTR d'ARNm de facteurs impliqués dans le maintien de l'intégrité de la MHB. En particulier, des sites de liaison de miR-9 sont présents sur les régions 3'UTR des ARNm fgf8, fgfrl, canopyl, her5 et her9. Par ailleurs, le profil d'expression de miR-9 était suggestif: il couvre une large portion du cerveau embryonnaire, à l'exception précisément de la MHB (Figure 2A). Ces premières données suggéraient que miR-9 pourrait jouer un rôle dans la restriction spatiale de la MHB, en inhibant à la fois la voie FGF et les facteurs Her.

La fonctionnalité des sites de fixation de miR-9 sur les ARNm codant des marqueurs de la MHB a pu être vérifiée in vivo chez l'embryon par des expériences de type «sensor assays » (Figure 2B). Le principe de ces tests consiste à injecter chez l'embryon au stade une cellule un ARNm comprenant la

Figure 1. Les deux activités de la frontière midbrainhindbrain boundary (MHB). Représentation schématique du cerveau de Danio rerio à mi-chemin du développement embryonnaire (vue latérale, côté antérieur vers la gauche). A. La MHB est la source d'un gradient de Fgf8 (orange) qui régionalise le mésencéphale et le rhombencéphale antérieur suivant l'axe antéropostérieur. B. La MHB exprime également les facteurs Her qui inhibent la neurogenèse, permettant le maintien local de progéniteurs (vert) alors que les régions voisines se différencient en neurones (violet). ant: région antérieure; dienc: diencéphale; post: région postérieure ; tél : télencéphale. 


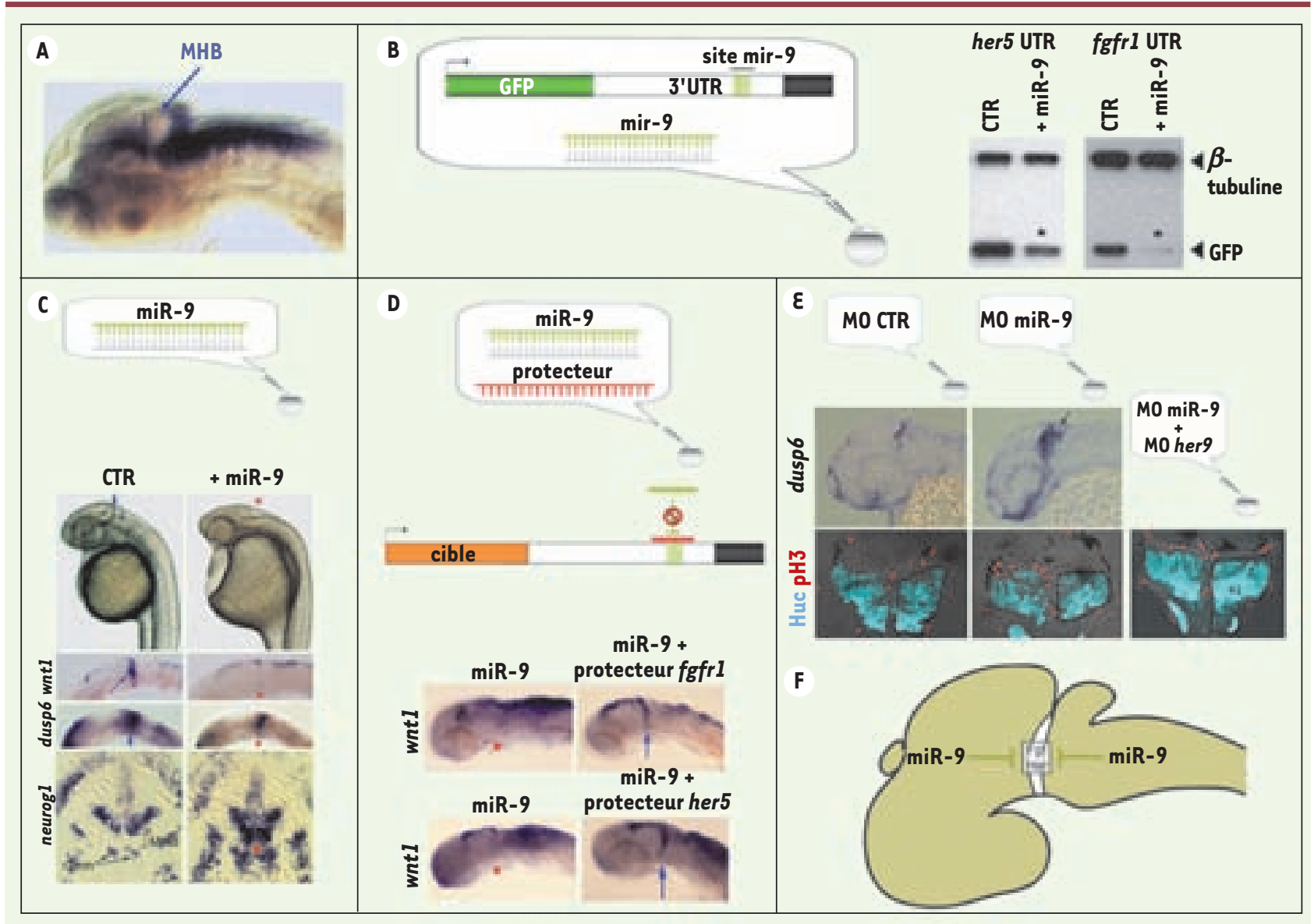

Figure 2. Analyse du rôle de miR-9 dans la régulation de la MHB. A. Expression de miR-9 chez un embryon de Danio zébré au stade 35 hpf (heures postfécondation) révélée par hybridation in situ (marquage bleu). miR-9 est exprimé dans le mésencéphale et le rhombencéphale mais est absent de la MHB (flèche bleue). B. Sensor assays. Un ARNm rapporteur comprenant la séquence codante de la GFP suivie de la région 3’UTR de la cible présumée (her5 ou fgfr1 dans ce cas) est injecté dans un embryon au stade une cellule, en combinaison avec le microARN miR-9 sous forme de duplex, ou d'un microARN contrôle (CTR). L'effet de miR-9 sur la traduction de la protéine indicatrice GFP est analysé par Western blot avec un anticorps spécifique de la GFP. Un anticorps anti-tubuline est également utilisé pour contrôler la charge du gel d'électrophorèse. Le Western blot révèle une quantité de GFP réduite par rapport au contrôle lorsque les embryons sont injectés avec miR-9. Cette expérience démontre que miR- 9 est capable d'interagir avec les régions 3'UTR des gènes her5 et fgfrl. C. Effet de la surexpression de miR-9. (en haut) Au stade 24 hpf, la MHB est visible morphologiquement chez les embryons injectés avec un microARN contrôle (flèche bleue) et non chez les embryons injectés avec un duplex miR-9 (astérisque rouge). ( $2^{e}$ et $3^{e}$ lignes) L'hybridation in situ avec des sondes spécifiques des gènes wntl et dusp6 montre que l'expression de ces gènes au niveau de la MHB au stade 12 somites (flèche bleue chez les embryons contrôles) est réduite chez les embryons injectés avec miR-9 (astérisque rouge). (4e ligne) Chez les embryons contrôles, le gène neurogeninl (neurogl) est exprimé spécifiquement par les précurseurs neuronaux du mésencéphale (vcc: ventro-caudal cluster) et du rhombencéphale ( $2 \mathrm{MN}$ : précurseurs de motoneurones du rhombomère 2). II est absent de la MHB (crochet). Chez les embryons injectés avec miR-9, une expression ectopique de neurogl est détectée au niveau de la MHB (astérisque rouge). D. Expérience de protection de cible. Les embryons au stade une cellule sont injectés avec miR- 9 et un morpholino protecteur de cible qui empêche la liaison de miR-9 sur la région 3'UTR des gènes fgfrl ou her5. Alors que les embryons injectés avec miR-9 présentent une absence d'expression de wntl au niveau de la MHB au stade 20 somites (astérisque rouge), les embryons co-injectés avec le protecteur de fgfrl ou her5 montrent une expression restaurée de ce gène au niveau de la MHB (flèche bleue). $\varepsilon$. Effet de l'inhibition de miR-9 par injection de morpholino (M0). (en haut) Coupes sagittales d'embryons injectés avec un MO contrôle (gauche) ou dirigé contre miR-9 (droite), hybridés avec une sonde spécifique du gène dusp6. L'injection du morpholino miR-9 conduit à un élargissement du domaine d'expression de dusp6 en comparaison avec les embryons contrôles. (en bas) Immunohistochimie sur coupes transversales du tube neural avec des anticorps dirigés contre la phosphohistone $\mathrm{H3}$ (pH3, rouge) qui marque les cellules en prolifération, et contre la protéine HuC, qui marque les neurones en différenciation (cyan). Chez les embryons injectés avec le M0 miR-9 (au milieu) la surface du tube neural marquée par l'anticorps anti-HuC est réduite par rapport aux embryons contrôles (à gauche). La co-injection d'un M0 dirigé contre

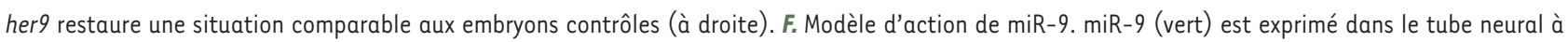
l'exception de la MHB, permettant de restreindre l'activité des gènes her et de la voie FGF à cette région. 
séquence codante d'une protéine indicatrice, la GFP (green fluorescent protein), suivie de régions 3'UTR de I'ARNm cible présumé. Si I'ARNm est bel et bien une cible de miR-9, dès lors la co-injection de miR-9 conduit à une diminution de la synthèse de GFP chez les embryons.

\section{Voies FGF et her : cibles de miR-9}

La surexpression de miR-9 chez l'embryon conduit à une perte morphologique de la MHB (Figure 2C) [6]. Elle est associée à une perte d'expression de marqueurs génétiques spécifiques de ce territoire, tel que wntl. L'absence d'expression du gène dusp6, cible directe de la voie FGF, signe par ailleurs une altération de cette voie chez ces embryons. Une induction précoce de neurogenèse au niveau de la MHB, zone où elle est normalement inhibée notamment grâce à l'action des gènes her5/9, est également observée. Ces données étant compatibles avec une action directe de miR-9 sur les transcrits de la voie FGF et des facteurs Her, nous avons voulu tester cette hypothèse grâce à des expériences dites de «protection de cible» [7]. Elles consistent à injecter en même temps que le microARN d'intérêt des oligonucléotides (morpholinos) bloquant l'accessibilité de celui-ci à ses sites sur les ARNm cibles (Figure 2D). L'injection de protecteurs spécifiques des sites de liaison de miR-9 présents sur les ARNm de fgfrl et her5 permet un sauvetage du phénotype de surexpression de miR-9. Cela démontre que l'effet de la surexpression de miR-9 est lié à son action sur ses cibles de la voie FGF et sur les facteurs Her.

Afin d'analyser le rôle endogène de miR-9, nous avons également réalisé des expériences de perte de fonction par injection d'un morpholino spécifiquement dirigé contre miR-9 (Figure 2E) [6]. La perte de fonction de miR-9 induit une augmentation de l'activité de la voie FGF ainsi que le montre l'augmentation de l'expression de dusp6. Par ailleurs, l'inhibition de l'activité de miR-9 conduit à une diminution de la neurogenèse au niveau de la MHB. Cette diminution peut être compensée par l'inhibition simultanée de her 9 , suggérant que la perte de fonction de miR-9 augmente l'activité des facteurs Her.
miR-9 coordonne l'action de la MHB

L'ensemble de ces données est en faveur d'une action simultanée de miR-9 sur la voie FGF et les facteurs $\varepsilon(s p l)$, permettant de restreindre spatialement à la fois l'activité organisatrice de la MHB et son réservoir de progéniteurs neuraux (Figure $2 F$ ). Elles font de miR-9 le premier facteur identifié comme responsable de la coordination de ces deux propriétés de la MHB. Cette étude illustre également que le Danio zébré est un modèle de choix pour l'étude du rôle des microARN au cours du développement chez les vertébrés, grâce aux différents outils d'analyses fonctionnelles disponibles chez cette espèce.

II semble vraisemblable que le rôle de miR-9 est conservé à l'échelle des vertébrés. Ainsi, des sites putatifs de miR-9 sont présents sur les régions 3'UTR de Hesl et de gènes de la voie FGF chez l'Homme. En outre, promouvoir la neurogenèse dans des cellules souches de mammifères en culture conduit à une induction transitoire de miR-9, et bloquer la fonction de miR-9 dans ce système expérimental entraîne une diminution de la différenciation neuronale [8], comme chez Danio. En revanche, miR-9 semble jouer un rôle très différent chez la drosophile. Chez cet organisme, miR$9 a$ est en effet exprimé dans les cellules épithéliales, chez lesquelles il inhibe un destin neuronal [9].

\section{MicroARN et développement : gardien des frontières?}

D’un point de vue plus général, d'autres études renforcent notre conclusion qu'un des rôles des microARN au cours du développement est d'affiner la position de frontières organisatrices, par une action au niveau post-transcriptionnel [13]. Ainsi miR-430 régule la balance de signalisation Nodal, régulant ainsi l'expansion du bouclier, tissu organisateur jouant un rôle majeur au cours de la gastrulation [7]. D'autres données suggèrent que des microARN régulent la position des frontières d'expression de gènes à homéodomaine Hox le long du tube neural [10]. Cependant, le rôle des microARN dans le système nerveux central ne se limite pas à la régulation des organisateurs. Même si les études fonctionnelles des microARN n'en sont qu'à leurs débuts, un large spectre de fonctions a d'ores et déjà été mis en évidence, allant de la spécification des différents types neuronaux à la régulation de la plasticité synaptique [11]. $\diamond$

A microRNA as a gatekeeper of the midbrain-hindbrain boundary

\section{REMERCIEMENTS}

Nos remerciements vont à Christoph Leucht, Christian Stigloher et Anja Folchert, qui ont réalisé les expériences résumées dans cet article, et aux autres membres de l'équipe, Magdalena Götz et Marion Wassef pour leurs suggestions et soutien au cours de ce travail. Notre équipe est financée par la fondation Volkswagen, le projet européen ZF-Models (LSHC-CT-2003-503466), l'Association Life Science 2005-01, l'ICM (Paris), le «Excellence Center for Protein Science » (Munich) et le « Helmholtz Impuls und Vernetzungsfonds».

\section{RÉFÉRENCES}

1. Bally-Cuif L, Hammerschmidt M. Induction and patterning of neuronal development, and its connection to cell cycle control. Curr Opin Neurobiol 2003; 13 : 16-25.

2. Meinhardt, H. Models of biological pattern formation : from elementary steps to the organization of embryonic axes. Curr Top Dev Biol 2008 ; 81 : 1-63.

3. Partanen, J. FGF signalling pathways in development of the midbrain and anterior hindbrain. J Neurochem 2007 ; 101 : 1185-93.

4. Stigloher C, Chapouton P, Adolf B, Bally-Cuif L. Identification of neural progenitor pools by $\varepsilon(\mathrm{Spl})$ factors in the embryonic and adult brain. Brain Res Bull 2008 ; 75 : 266-73.

5. Boyd SD. Everything you wanted to know about small RNA but were afraid to ask. Lab Invest 2008; 88 : 569-78.

6. Leucht C, Stigloher C, Wizenmann A, et al. MicroRNA-9 directs late organizer activity of the midbrain-hindbrain boundary. Nat Neurosci $2008 ; 11: 641-8$.

7. Choi WY, Giraldez AJ, Schier AF. Target protectors reveal dampening and balancing of Nodal agonist and antagonist by miR-430. Science $2007 ; 318: 271-4$.

8. Krichevsky AM, Sonntag KC, Isacson 0, Kosik KS. Specific microRNAs modulate embryonic stem cell-derived neurogenesis. Stem Cells $2006 ; 24: 857-64$.

9. Li Y, Wang F, Lee JA, Gao FB. MicroRNA-9a ensures the precise specification of sensory organ precursors in Drosophila. Genes Dev 2006 ; 20 : 2793-805.

10. Woltering JM, Durston AJ. MiR-10 represses HoxBla and HoxB3a in zebrafish. PLoS One 2008 ; 3 : el396.

11. Gao FB. Posttranscriptional control of neuronal development by microRNA networks. Trends Neurosci $2008 ; 31: 20-6$.

12. Hartmann C, Corre-Menguy F, Boualem A, et al. Les microARN : une nouvelle classe de régulateurs de l'expression génique. Med Sci (Paris) 2004 ; 20 : 894-8.

13. Peaucelle A, Laufs P. Des casques bleus chez les plantes: un microARN, miR164, stabilise les frontières dans le méristème. Med Sci (Paris) 2006 ; 22 : 796-8. 